\title{
Implementation of Fuzzy Logic with High Security Registration Plate (HSRP) for Vehicle Classification and Checking in Toll- Plaza
}

\author{
V. Sathya ${ }^{1}$ and J. Abdul Samath ${ }^{2}$ \\ ${ }^{1}$ AP, Department of Computer Applications, MGR College, Hosur, India \\ sathyanakar@gmail.com \\ ${ }^{2}$ Head, Department of Computer Applications, SRIT, Coimbatore, India \\ abdul_samath@yahoo.com
}

\begin{abstract}
In Automobile Industries, to use of High Security Registration plate (HSRP) is still a challenging problem. There are more options to misuse the vehicle and exchange its engine, chassis, gear box, axle etc., In an existing system, the Regional Transport Office (RTO) only determine an abstract of the vehicle and its owner. The vehicles are classified using piezo sensor and inductive loop systems. The toll-plaza is used only collected fees from the vehicles for maintain the quality roads. There are no authorized agencies allotted to identify the vehicle checking and no possibilities to control the vehicle overloading. The proposed system, toll-plaza will be act as a multi-plaza. Vehicles are classified with weight and speed. Then it is checking in toll-plaza either passed or checked. In this paper, The system uses illumination (such as Infrared) and a camera to take the image of the front or rear of the vehicle, then an extracts the plate information. This data is used for enforcement and it can be used to open a gate if the vehicle is checked with RTO data in toll-plaza. In vehicle checking, we develop new rules using a fuzzy logic to improve the performance. The features of this system are implemented in the upgrading vehicles only. It is used to control the overloading to maintain road safety and to identify the theft vehicle to reduce the crime and terrorism. As Bharat Stage Emission (BSE) standard vehicles are implemented in India very aggressively. The emission standard vehicles are serviced only in authorized service centre not for doing and end root machines.
\end{abstract}

\section{Keywords}

HSRP (High Security Registration Plate), Fuzzy logic, vehicle classification, vehicle checking,

Toll-plaza, overloading.

\section{INTRODUCTION}

In India High Security Registration Plate (HSRP) is not implemented in all States. So as per Supreme Court instruction only few states to be implement this project. At earlier, UTSAV is the first Company in India to get the approval from Automotive Research Association of India (ARAI) for manufacturing HSRP. Now Government approved more than 15 agencies manufactured this plate. It's get the order from the concerned Government states and to be manufacturing the plate and supply to the public. The HSRP plate consists of new holograms with our old registration authority policies. The Ministry of Road Transport \& Highways, Indian Government has amended Rule 50 of Central motor Vehicle Rules 1989 to standardize the pattern of displaying registration mark throughout India.

Sundarapandian et al. (Eds): CoNeCo,WiMo, NLP, CRYPSIS, ICAIT, ICDIP, ITCSE, CS \& IT 07, pp. 297-306, 2012. (C) CS \& IT-CSCP 2012

DOI : $10.5121 /$ csit.2012.2428 
Under the existing system, where all records are manually created and maintained, it is difficult to compile national data of motor vehicles. HSRP will help in creating computerized data of old registered vehicles and new vehicles at places where computerized records are not maintained at present. With the networking of all RTOs, it will become easy to prepare national data of motor vehicles. Recently, traffic congestion has become a serious problem. However, the construction of new roads alone is not the solution to effective traffic management [1,2]. Vehicle classification data can serve as the fundamental data for planning new road constructions, establishing road maintenance policies, and calculating travel times.

Many studies have been conducted to identify vehicles using various traffic sensors, such as microwave, ultrasonic, inductive loop, video image, and vehicle sound sensors [1,3]. One of the most commonly used technologies for vehicle classification is the combined loop and piezoelectric sensor system $[2,4]$. In this loop/piezo sensor fusion detector, the vehicle length information is one of the most important data [1,2]. Therefore, obtaining accurate vehicle length data is critical to obtain accurate vehicle classification results.

Fuzzy algorithms have been successfully applied to a variety of industrial applications, including automobiles, autonomous vehicles, chemical processes, and robotics [5,6]. In the loop/piezo detector, there is no exact mathematical relationship between the vehicle length and speed, or between the length and shape. However, a heuristic knowledge of how the vehicle speed or shape may have an influence on the measured length is available. This heuristic knowledge can be expressed well in terms of a fuzzy logic using the so-called fuzzy IF-THEN rules [9]. This paper discusses improving the vehicle classification performance of loop/piezo detectors, by the use of fuzzy rules.

License Plate Recognition (LPR) is an image processing technology also used to identify vehicles by their license plates. This technology is gaining popularity in security and traffic installations. The technology concept assumes that all vehicles already have the identity displayed (like HSRP plate) so no additional transmitter or responder is required to be installed on the car. The system uses illumination (such as Infra-red) and a camera to take the image of the front or rear of the vehicle, then image processing software analyzes the images and extracts the plate information. This data is used for enforcement, data collection, and it can be used to open a gate if the car is authorized or keep a time record on the entry or exit for automatic payment calculations. The LPR system significant advantage is that the system can keep an image record of the vehicle which is useful in order to fight crime and fraud. An additional camera can focus on the driver face and save the image for security reasons. Additionally, this technology does not need any installation per car.

This paper is organized as follows. Section 2 describes the proposed model of vehicle in tollplaza with HSRP and Licence Plate recognition. Section 3 describes developed vehicle classification and toll-plaza checking using fuzzy rules. Section 4 describes prevention of overloading. Section 5 illustrates the experimental results on different testing sets. Finally a conclusion is presented in section 6.

\section{PROPOSED MODEL OF VEHICLE WITH HSRP AND LP RECOGNITION}

HSRP to avoid theft of vehicles and misuse in terror related incidents. It is mandated by Govt. of India to be affixed on all new vehicles from Oct 31st, 2007. All Existing vehicles will have to switch over to HSRP's within 2 years from date of implementation, now the State Governments have to implement this policy in the respective States at the earliest. But, now only a few states have implemented with this law. And the other states are in the process to finalize the tenders. The salient features of HSRP are shown in Figure. 1. 
The standard formats of HSRP are

2wheelers $\quad-200 \times 100 \mathrm{~mm} \& 285 \times 45 \mathrm{~mm}$ (Font)

Car/LMVs $\quad-340 \times 200 \mathrm{~mm} \& 500 \times 120 \mathrm{~mm}$

Heavy Vehicles - 340 x $200 \mathrm{~mm}$

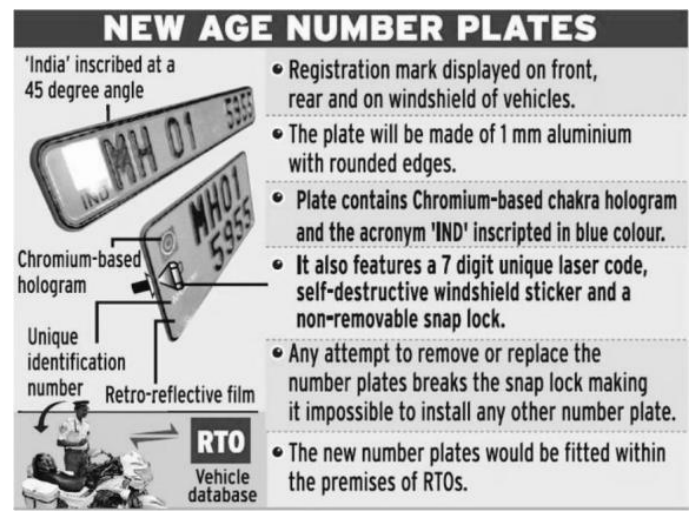

Figure.1. Features of High Security Registration Plate

License Plate units are based on images of the front and/or rear plates. For example of Figure. 2 shows, the car with the plate number: WB04B5520, is just exiting from the secured area and is picked up by the LPR system which identifies it as an authorized car and opens the gate. Where

o The first two letters of the registration plate represent the State in which the vehicle is registered.

o The next two digit numbers are the sequential number of a district. Due to heavy volume of vehicle registration, the numbers were given to the RTO offices of registration as well.

o The third part is a four digit numbers unique to each plate. A letter is prefixed when the four digit number runs out and then letters and so on.

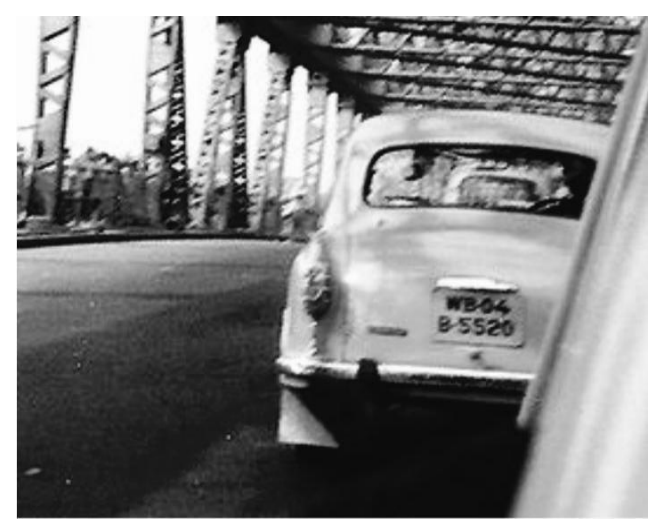

Figure 2. License plate on the back of a car in Calcutta

A typical image captured by the Infra-red illumination and camera. The image information is read by the LPR unit and is analyzed and recognized automatically. Note that the image is monochrome since the infra-red spectrum is above the normal colour spectrum. The LPR 
systems are Country specific and are adapted to the Country where they are installed and used. The given image of the front side of the car, shown in a typical format, is composed of 256 grey levels ranging from black (grey level 0) to white (grey level 255). For a typical format there are 768 X 288 pixels, or about 0.2 Million elements. This vast amount of information is processed by the recognition software in order to automatically locate and read the plate. Early LPR systems suffered from a low recognition rate, lower than required by practical systems. The external effects (sun and headlights, bad plates, wide number of plates types) yielded low quality systems. LPR systems normally consist of the following units:

- Camera - that take the images of the car (front or rear side)

o Illumination - a controlled light that can bright up the plate, to allow day and night operation. In most cases the illumination is Infra-Red which is invisible to the driver.

o Database - the events are recorded on a local database or transmitted over the network. The data includes the recognition results and (optionally) the vehicle or driver-face image file.

\section{VeHICle Classification \& CheCKING In TOLl-PlaZa USING FUZZY RULES}

This section consists of two parts. First one is vehicle classification using piezo/loop sensor systems. Second one is Toll-plaza checking using illumination (such as infra-red) and a camera. Both are developed in fuzzy rules.

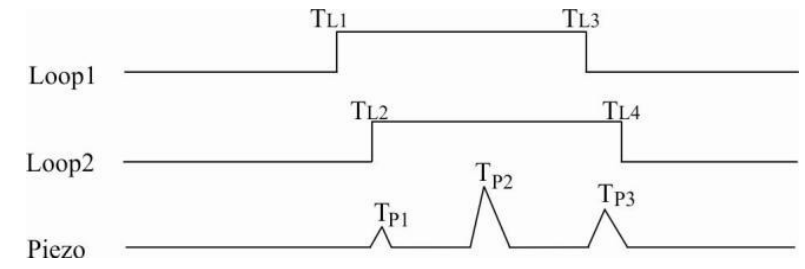

Figure 3. Typical output waveform of two inductive loops and one piezoelectric sensor (3-axle vehicle)

The inductive loop coil is used to detect the presence of a passing vehicle by sensing the inductance change in the loop coil, and the piezoelectric sensor is used to detect a passing vehicle by the pressure generated from tires. The system configuration can be either two inductive loops and one piezoelectric sensor, or one inductive loop and two piezoelectric sensors [2,8]. Here, this system used with two inductive loops and one piezoelectric sensor.

However, the typical output of the inductive loop is a digital on-off signal, and the output of the piezoelectric sensor is an analog signal as shown in Fig. 3. From the time data (TL1, TL2, TL3, TL4, TP1, TP2, and TP3 ), the vehicle speed and length, distance between axles can be calculated. The total vehicle weight as well as individual axle weight information can also be obtained by processing the piezoelectric sensor signal.

The Figure $4 \& 5$ shows configuration of the developed algorithm. The dashed box represents a newly developed fuzzy logic block $1 \& 2$. Here the both fuzzy rules are interpreted which have some of linguistic values are as follows:

$$
\begin{aligned}
& \text { Speed }=\{\operatorname{slow}(\mathrm{S}), \operatorname{medium}(\mathrm{M}), \operatorname{fast}(\mathrm{F})\}, \\
& \text { Weight }=\{\operatorname{very} \operatorname{light}(\mathrm{VL}), \operatorname{light}(\mathrm{L}), \operatorname{medium}(\mathrm{M}), \operatorname{heavy}(\mathrm{H})\},
\end{aligned}
$$




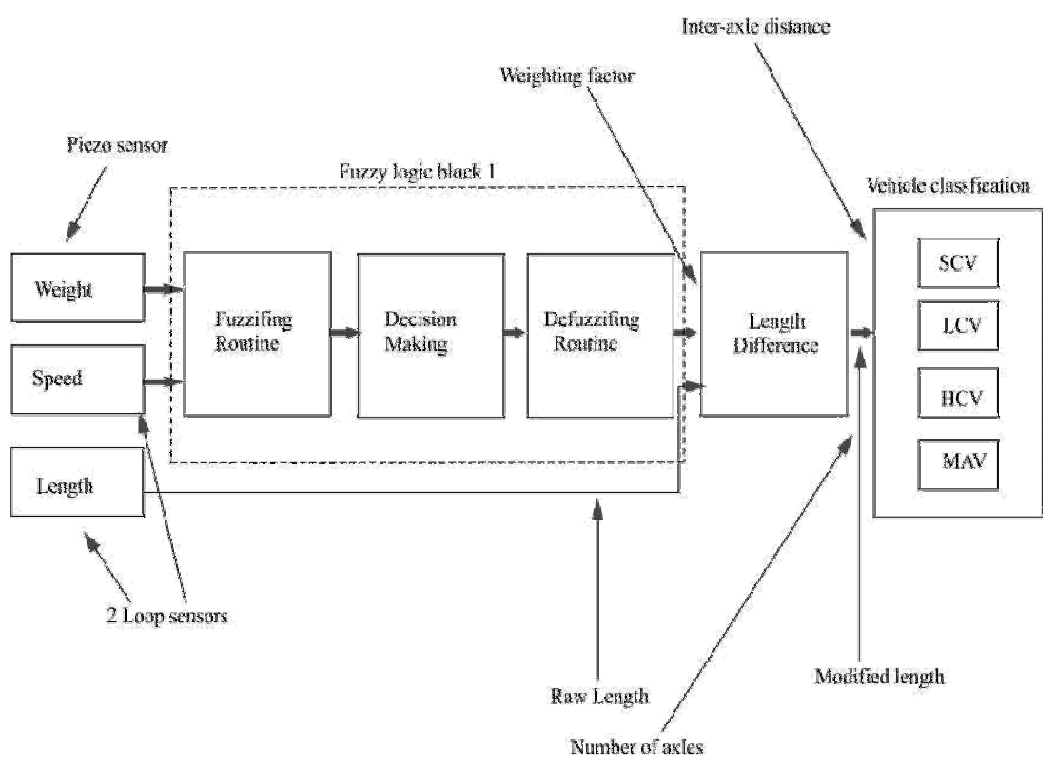

Figure 4. The configuration of the fuzzy logic block I

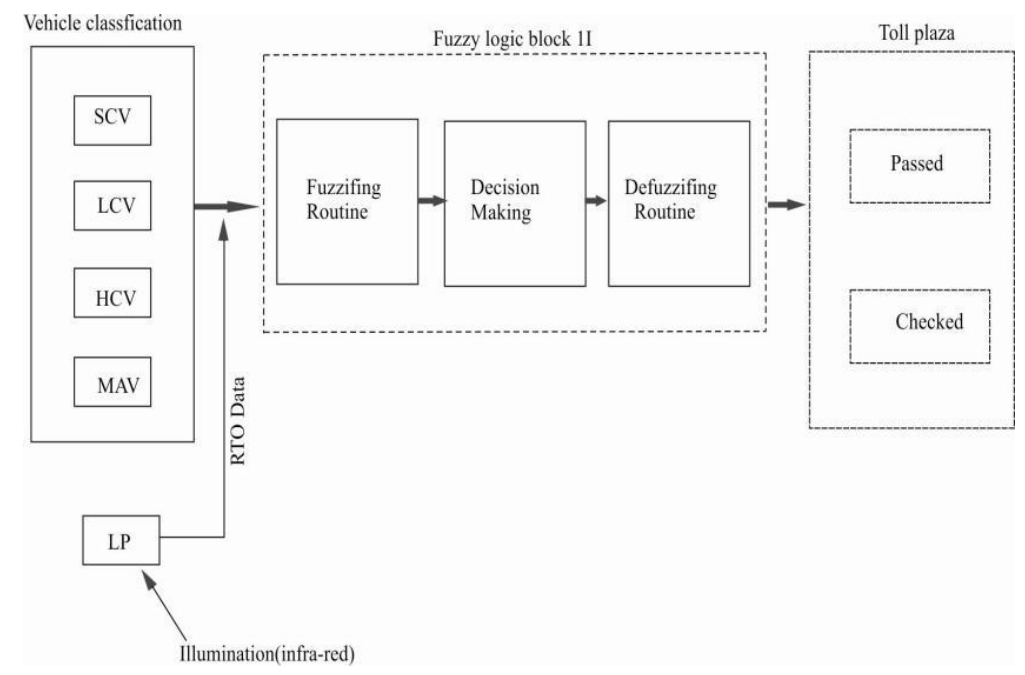

Fig.5. The configuration of the fuzzy logic block II

Ldifference $=\{$ negative big(NB), negative small(NS), zero(ZE), positive small(PS), positive $\operatorname{big}(\mathrm{PB})\}$

Vehicle classification $($ vclass $)=\{$ small commercial vehicle $(\mathrm{SCV})$, light commercial vehicle(LCV), heavy commercial vehicle(HCV), multi Axle vehicle(MAV)\}

Vehicle status $=\{$ passed, checked $\}$ 

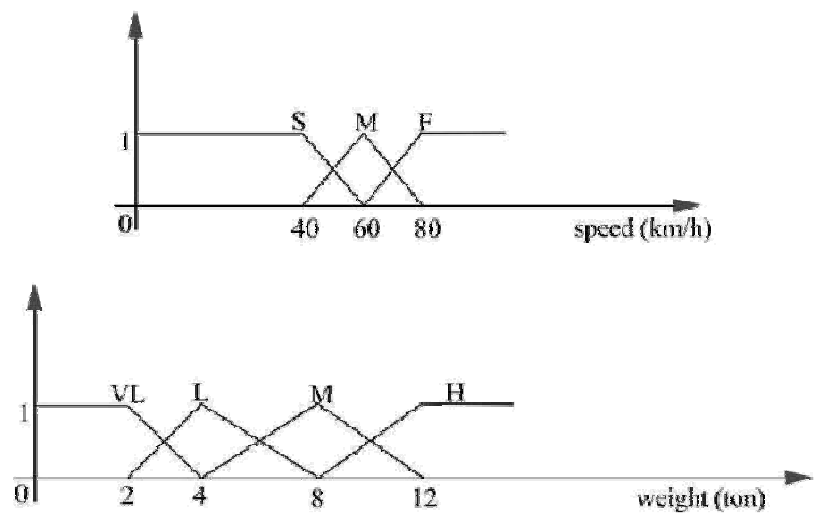

Figure 6. Membership functions for input of vehicle speed \& weight for Fuzzy block I

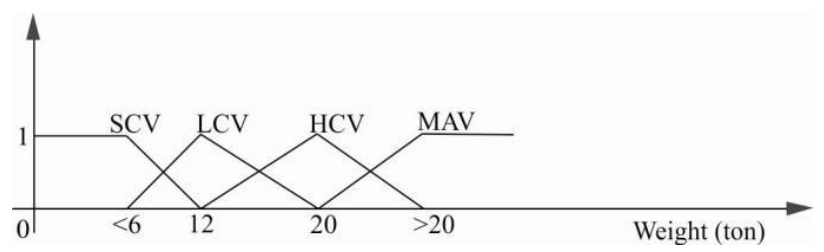

Figure 7. Membership functions for input of vehicle classification for Fuzzy block II

Figure $6 \& 7$ represented as membership functions for inputs of fuzzy block I \& II. Normally, the represented vehicles are categorized basis of weight are as follows:

SCV (Small Commercial Vehicle/Car) - below 6 tones

LCV (Light Commercial Vehicle) - 6 to 12 tones

HCV (Heavy Commercial Vehicle) - 12 to 20 tones

MAV (Multi-Axle overloaded Vehicles) - 20 and above

Based on the rule base, the output of fuzzy system is computed through two steps: an inference step and a defuzzification step. Among the various mechanisms representing the meaning of IFTHEN rules in the inference step, the Mamdani implication is used in this paper, which is one of the most widely used implications in applications of fuzzy logic [5,7,9]. In the defuzzification step, center of gravity method (COG) is used. Each linguistic value is represented by an appropriate membership function. The fuzzy rule base is an IF-THEN linguistic rule using the fuzzy input and output sets. The fuzzy rules of vehicle classifications are as follows: 
IF weight is very light and speed is slow, THEN ldifference is zero

IF weight is very light and speed is medium, THEN ldifference is negative small

IF weight is very light and speed is fast, THEN ldifference is negative big

IF weight is light and speed is slow, THEN ldifference is positive small

IF weight is light and speed is medium, THEN ldifference is zero

IF weight is light and speed is fast, THEN ldifference is negative small

IF weight is medium and speed is slow, THEN ldifference is positive big

IF weight is medium and speed is medium, THEN ldifference is positive small

IF weight is medium and speed is fast, THEN ldifference is zero

IF weight is heavy and speed is slow, THEN ldifference is positive big

IF weight is heavy and speed is medium, THEN ldifference is positive big

IF weight is heavy and speed is fast, THEN ldifference is positive small

The modified vehicle length is calculated as

Length difference $=$ measured length $\times(1+($ weighting factor/100 $))$

where measured length is calculated using the raw outputs of two inductive loops, and weighting factor is the output of the fuzzy logic block. The modified length is the input to the vehicle classification block, and the final classification result is generated. The developed fuzzy rules of vehicle checking in toll-plaza are as follows:

IF vclass is scv and lpdata is positive, THEN vehicle is passed

IF vclass is scv and lpdata is negative, THEN vehicle is checked

IF vclass is lcv and lpdata is positive, THEN vehicle is passed

IF vclass is lcv and lpdata is negative, THEN vehicle is checked

IF vclass is hov and lpdata is positive, THEN vehicle is passed

IF vclass is hev and lpdata is negative, THEN vehicle is checked

IF vclass is mv and lpdata is positive, THEN vehicle is passed

IF vclass is mv and lpdata is negative, THEN vehicle is checked

While the vehicle approaches the toll gate, the LPR unit automatically reads the license plate registration number, compares to a predefined list and opens the gate if there is a match. Otherwise it is inform to owner of the vehicle, RTO and local police station. The concept is that all vehicles already have the identity displayed (HSRP plate). So no additional transmitter or responder is required to be installed on the car.

The most popular vehicle sensor like magnetic loop detector senses the car and its presence is signal to the LPR unit. It is activates the illumination (invisible Infra-red in most cases) and takes pictures of the front or rear plates from the LPR camera shown at the left side of the gate. The images of the vehicle include the plate and the pixel information is read by the LPR unit's image processing hardware (the frame grabber). Fig. $8 \& 9$ represented as membership functions for outputs of fuzzy block I \& II. 


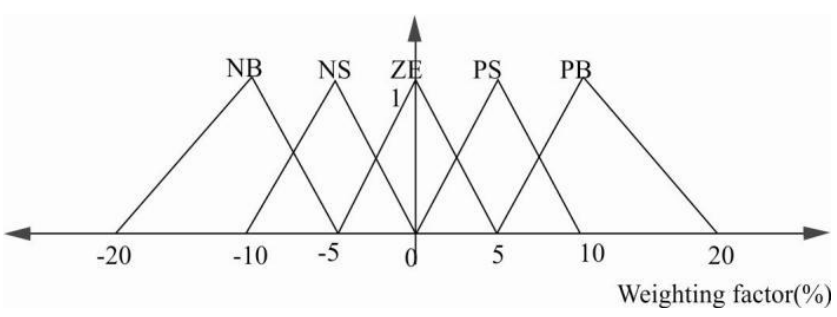

Figure 8. Membership functions for output of Fuzzy block I

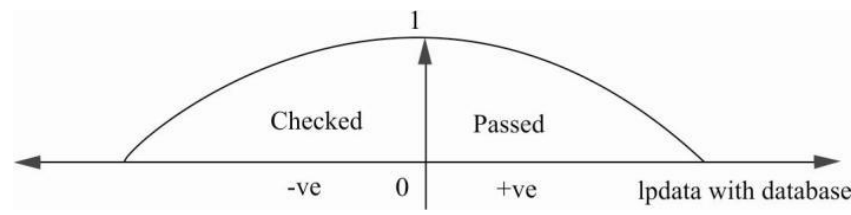

Figure 9. Membership functions for output of Fuzzy block II

In Toll-plaza (Figure.10), the vehicle number is used to calculate the travel fee in a toll-road. That is, the plate is read when the vehicle enters the toll lane and presents a pass card. The information of the vehicle is retrieved from the database and compared against the pass information. In case of fraud the operator is notified.

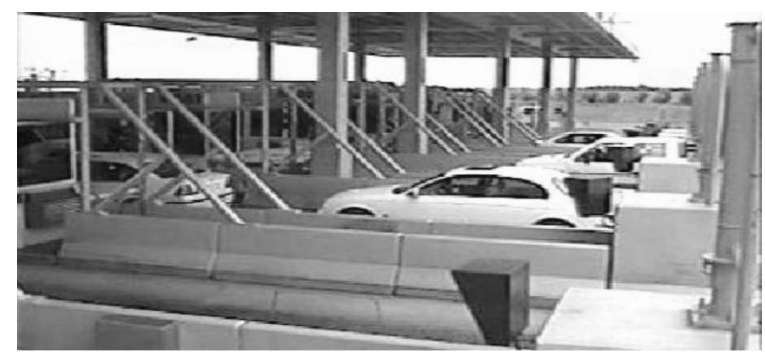

Figure.10. Queue of vehicle in toll-plaza

\section{PREVENTION OF OVERLOADING}

Overloading causes more problems other than the obvious injury or thrashing of life to the driver, loading/unloading staff, other road users or the public.

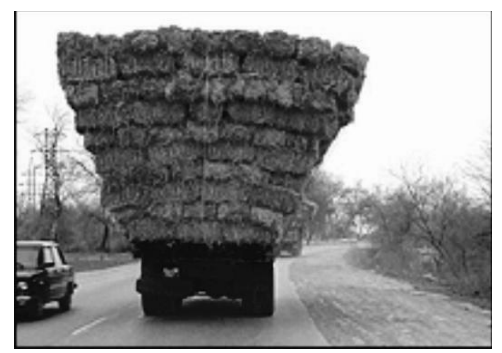

Figure 11. Prevention of overloading 
The resultant problems caused through overloading any vehicles (shown in Figure11) are:

- Smash to roads, bridges and concretes etc.

- Drivers are inability to shove and brake correctly.

- To made tires failure.

- Causing vehicle to be unbalanced.

Overloading is not only a problem for heavy goods vehicle, it is equally a problem of smaller vehicles, such as vans, cars and passenger carrying vehicles. So, the proposed system used as,

- To maintain road safety.

- To identify the theft vehicle.

- To reduce the crime and terrorism

- To identify the vehicle is original or not

- To control the speed limitations.

\section{EXPERIMENTAL RESULTS}

The developed algorithm is tested in an experimental site of the vehicle shown in Figure 12. In our experiments, vehicle classification algorithm [2,9] and the newly developed fuzzy algorithms are tested together for performance comparison.

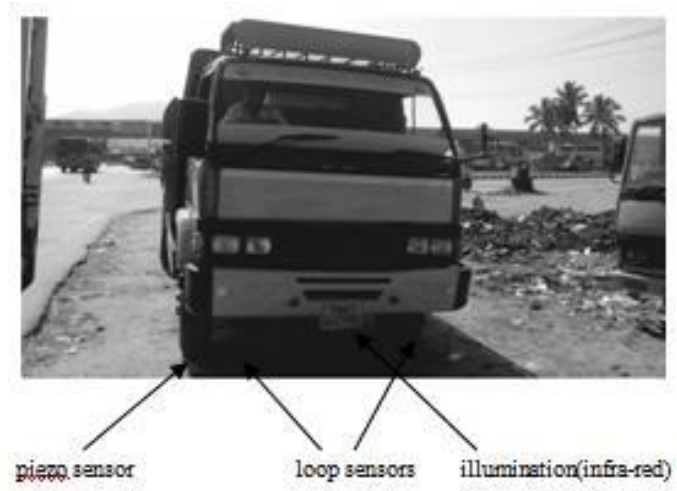

Figure 12. Experimental site of the vehicle

In accordance with Ministry of Road Transport and Highways in India, vehicles are categorized into 4 groups. The representative vehicles of each category are shown in Fig. 13 as follows: Group A (Small Commercial Vehicle/Car), Group B (Light Commercial Vehicle), Group C (Heavy Commercial Vehicle), Group D (Multi-Axle overloaded vehicles). Compared to existing system, error rate is decrease to least percentage only. Total number of passing vehicles in the experiment was 247 . The passing vehicles are classified into 4 categories, and the results of vehicle classification and checking errors are generated. 


\section{CONCLUSION}

In this paper, a new vehicle checking technology using fuzzy rules are developed in toll-plaza. That is, the vehicle weight and speed are used as the inputs to the fuzzy logic block I. The output of the fuzzy logic block I is a weighting factor to modify the vehicle length calculated using the raw sensor outputs. The modified length is the input to the vehicle classification block. This classification and license plate recognition number with pre-determined data are used to fuzzy logic block II. The output is generated, to the vehicle is either passed or checked in toll-plaza. Experimental results show that the proposed classification algorithm using the fuzzy logic significantly reduces the errors. This is used to identify the theft vehicle for reduce the crime and to avoid terrorism for misuse in terror related incidents. This is also used to control the overloading to maintain road safety. This technology is gaining popularity in security and traffic installations.

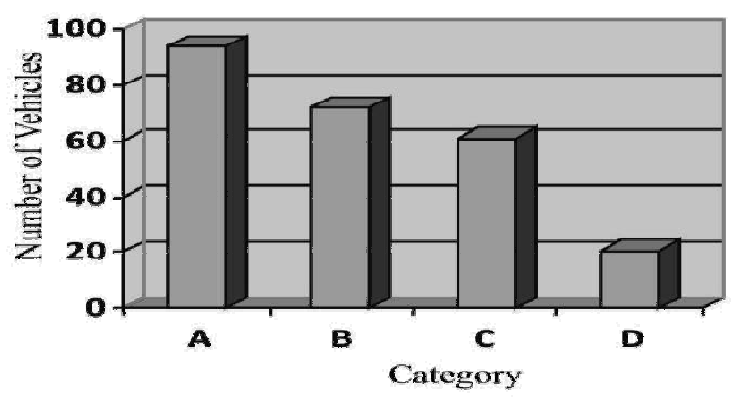

Figure 13. Number of passing vehicles in each group

\section{REFERENCES}

[1] Coifman, B., "Vehicle Reidentification and Travel Time Measurement in Real-time on Freeways Using the Existing Loop Detector Infrastructure," Transp. Res. Rec. 1643, pp. 181-191.

[2] Kim, S.W., Y. Eun, H. Kim, J.I. Ko, W.J. Jung, Y.G.. Choi, Y.G.. Cho and D. Cho, Performance Comparison of Loop/Piezo and Ultrasonic Sensor-based Detection Systems for Collecting Individual Vehicle Information,” Proc. 5th World Congr. Intell.Transp. Syst.,Seoul, Korea.

[3] Yung, N.H.C., K.C. Chan and A.H.S. Lai, "Vehicletype Identification through Automated Virtual Loop Assignment and Block-based Direction Biased Motion Estimation,” Proc. IEEE/IEEJ/JSAI Int. Conf. Intell. Transp. Syst., Tokyo, Japan.

[4] Kim, S.W., J.I. Ko, H. Kim, I. Cho and D. Cho, "A New Loop-detector Circuit for Improving lowspeed Performance," Proc. 6th World Congr. Intell. Transp. Syst., Toronto, Canada.

[5] Passino, K.M. and S. Yurkovich, Fuzzy Control, Addison-Wesley, Reading, MA.

[6] Lai, M., M. Nakano and G. Hsieh, "Application of Fuzzy Logic in the Phase-Locked Loop Speed Control of Induction Motor Drive,” IEEE Trans. Ind. Electron., Vol. 43, No. 6, pp. 630-639.

[7] Driankov, D., H. Hellendoorn and M. Reinfrank, An Introduction to Fuzzy Control, Springer-Verlag, Berlin, Heidelberg.

[8] Newton, W.B., B.A. Frith and I.A. Barbour, "Use of Weigh-in-motion Systems to Aid the Selection of Goods Vehicles for Enforcement Weighing," Proc. IEE 2nd Int. Conf. Road Traffic Monitoring, London, U.K., pp. 24-28.

[9] Wang, L.X., A Course in Fuzzy Systems and Control, Prentice-Hall, Upper Saddle River, NJ

[10] Jang J-S and N. Gulley, Fuzzy Logic Toolbox, The MathWorks Inc, Natick, MA. 